\title{
Philosophiques
}

\section{L'argument du sens commun et la " persécution des scientifiques ». Collingwood contre Moore}

\section{Laurent Jaffro}

Volume 36, numéro 1, printemps 2009

L'idéalisme britannique

URI : https://id.erudit.org/iderudit/038013ar

DOI : https://doi.org/10.7202/038013ar

Aller au sommaire du numéro

\section{Éditeur(s)}

Société de philosophie du Québec

ISSN

0316-2923 (imprimé)

1492-1391 (numérique)

Découvrir la revue

Citer cet article

Jaffro, L. (2009). L’argument du sens commun et la « persécution des scientifiques ». Collingwood contre Moore. Philosophiques, 36(1), 131-147. https://doi.org/10.7202/038013ar
Résumé de l'article

En s'appuyant surtout sur An Essay on Philosophical Method (1933) et sur The New Leviathan (1942), cet article expose les principaux arguments employés par R. G. Collingwood contre le recours au sens commun qu'utilisait G. E. Moore. Selon The New Leviathan, le recours au sens commun comme à une protection contre le scepticisme ou l'idéalisme conduit à la " persécution des scientifiques " et à l'« obscurantisme ". On peut tenir ce point de vue pour exagéré. Toutefois, si l'on examine la construction de l'argument du sens commun, la pertinence de la critique de Collingwood apparaît. Cela n'empêche pas celui-ci d'utiliser la notion de sens commun, compris comme un ensemble de croyances fondamentales. Il n'y a aucune contradiction en l'espèce, pourvu qu'on distingue la notion du sens commun de l'argument du sens commun. 


\title{
L'argument du sens commun et la «persécution des scientifiques ». Collingwood contre Moore
}

\author{
LAURENT JAFFRO \\ Université Blaise-Pascal, Clermont-Ferrand
}

\begin{abstract}
RÉSUMÉ. - En s'appuyant surtout sur An Essay on Philosophical Method (1933) et sur The New Leviathan (1942), cet article expose les principaux arguments employés par R. G. Collingwood contre le recours au sens commun qu'utilisait G. E. Moore. Selon The New Leviathan, le recours au sens commun comme à une protection contre le scepticisme ou l'idéalisme conduit à la «persécution des scientifiques » et à l'«obscurantisme». On peut tenir ce point de vue pour exagéré. Toutefois, si l'on examine la construction de l'argument du sens commun, la pertinence de la critique de Collingwood apparaît. Cela n'empêche pas celui-ci d'utiliser la notion de sens commun, compris comme un ensemble de croyances fondamentales. Il n'y a aucune contradiction en l'espèce, pourvu qu'on distingue la notion du sens commun de l'argument du sens commun.
\end{abstract}

\begin{abstract}
Drawing mainly on An Essay on Philosophical Method (1933) and on The New Leviathan (1942), this paper sets out R. G. Collingwood's main arguments against G. E. Moore's appeal to common sense. According to The New Leviathan, the recourse to common sense as a safeguard against scepticism or idealism leads to "scientific persecution" and "obscurantism". That view might be considered as excessive. However, after a close examination of the structure of the argument from common sense, Collingwood's critique appears to be relevant. This does not prevent him from using the notion of common sense, understood as a set of basic beliefs. There is no contradiction here, provided that we distinguish the notion of common sense from the argument from common sense.
\end{abstract}

Depuis l'école écossaise du sens commun, à la fin du XviII ${ }^{e}$ siècle, jusqu'à la philosophie de G. E. Moore - et sans doute bien au-delà —, on a recours au sens commun contre l'idéalisme subjectif ou le scepticisme. En dépit des différences entre les formes qu'a pu prendre ce recours chez tel ou tel, les philosophes du sens commun s'accordent à considérer qu'on trouve dans le sens commun à la fois un point de départ pour la philosophie et un gardefou contre ce qu'ils estiment être des pathologies théoriques.

Cette étude a pour but de présenter deux arguments différents que Collingwood a opposés au recours philosophique au sens commun, et qu'il a élaborés dans le contexte de son combat contre ce qu'il appelle la "philosophie analytique ", notamment représentée par G. E. Moore.

Je commence par reconstituer une forme générale de l'argument du sens commun dont on peut prétendre qu'elle est commune à des philosophes divers, de Reid à Moore. Il est en effet nécessaire d'identifier préalablement l'objet exact de cette discussion. Ensuite, j'expose les deux arguments de Collingwood, dont la portée est inégale, mais qui me semblent intéressants parce qu'ils pointent la complaisance et la misologie qui caractérisent souvent 
le recours philosophique au sens commun, par exemple chez les auteurs qui aujourd'hui (dans une inspiration prétendument wittgensteinienne ou reidienne) dénoncent le scepticisme comme une comédie hypocrite et indigne de la vraie philosophie. Je ne situerai pas, sinon allusivement, la place de cette discussion dans l'ensemble de l'œuvre de Collingwood, car je m'intéresserai plutôt à la chose même qu'est le recours philosophique au sens commun. Un des buts de cet exposé est de rendre sensible la différence entre ce que j'appellerai l' "argument du sens commun" et la "notion du sens commun ", et de montrer comment cette distinction peut contribuer à renforcer l'argumentation de Collingwood.

\section{Que doit être le recours philosophique au sens commun pour pouvoir constituer un argument correct?}

Cette question ne présuppose pas que l'argument soit correct, mais cherche à déterminer quelles conditions il doit remplir pour ne pas être simplement impossible. Il faut commencer par déterminer ce qu'on entend ici par "argument du sens commun». Ce n'est pas une mince affaire, car le recours philosophique au sens commun est ambigu, comme cela a été souvent souligné. Il faut essayer d'en proposer des formulations.

Formulation 1: Soit une thèse philosophique $\mathrm{T}$ en faveur de laquelle un philosophe argumente. Avoir recours au sens commun contre cette thèse $T$, c'est montrer que $T$ contredit un principe de sens commun ou bien que les conséquences ou les implications de $\mathrm{T}$ contredisent un principe de sens commun.

Cette présentation du recours au sens commun est cependant incomplète, car elle laisse dans l'ombre une clause de justification $\mathrm{C}$ qu'il faut expliciter.

Formulation 2: Avoir recours au sens commun contre T, c'est montrer que $\mathrm{T}$ (soit par elle-même soit par ses conséquences) contredit un principe de sens commun alors que $\mathrm{C}$ : on a de bonnes raisons de rejeter une thèse qui contredit le sens commun.

L'ambiguiité fondamentale réside dans C. Cette clause de justification reste souvent implicite, et alors l'ambiguité n'est pas décelée. Si T contredit les indications du sens commun, cela semble suffire à justifier qu'elle soit écartée, et en général on ne dit pas plus que «c'est contraire au sens commun ». Mais en vérité on présuppose $\mathrm{C}$, à savoir qu'on est justifié d'écarter une thèse parce qu'elle est contraire au sens commun. $\mathrm{C}$ revient à affirmer qu'une thèse qui contredit le sens commun doit être rejetée. Or il y a une ambiguité dans $\mathrm{C}$. On peut comprendre $\mathrm{C}$ ou comme $\mathrm{C} 1$ ou comme $\mathrm{C} 2$ :

C1: Une thèse qui contredit le sens commun doit être rejetée en tant qu'elle contredit une vérité de sens commun, c'est-à-dire une proposition qui est vraie parce qu'elle est communément admise. 
C2: Une thèse qui contredit le sens commun doit être rejetée en tant qu'elle contredit une vérité de sens commun, c'est-à-dire une proposition qui est communément admise parce que sa vérité ne fait aucun doute.

Seule l'interprétation de C comme C2 est susceptible d'être correcte je veux dire, n'est pas manifestement sophistique.

J'emprunte cette manière de présenter ce qu'est un recours correct au sens commun à Noah Lemos:

Bien que la tradition du sens commun soutienne effectivement que certaines propositions du sens commun sont pour nous justifiées épistémiquement, elle ne fait pas sienne la conception selon laquelle elles sont pour nous justifiées épistémiquement en vertu $d u$ fait qu'elles sont des propositions de sens commun ou en vertu du fait qu'elles sont profondément ancrées et très largement répandues ${ }^{1}$.

Pour Lemos, cette conception est commune à Reid, à Moore et à d'autres auteurs du $\mathrm{xx}^{\mathrm{e}}$ siècle. Cependant, Lemos considère qu'un recours philosophique au sens commun de ce type est correct (à la seule condition que la proposition de sens commun soit vraie), alors qu'il me semble qu'on a identifié ainsi seulement un recours philosophique au sens commun qui n'est pas manifestement sophistique.

$\mathrm{Si}$ on interprète $\mathrm{C}$ comme $\mathrm{C} 1$ et si on soutient un recours au sens commun de ce style, il est manifeste qu'on autorise le dogmatisme et l'obscurantisme (qui consistent à donner raison par principe au sens commun) et, en passant, on ne se prémunit pas contre une faute de raisonnement. Il y a un rapport très étroit entre cette question de l'interprétation de la clause de justification et une faute de raisonnement qu'on a souvent pointée dans les recours insuffisamment réfléchis au sens commun.

On peut présenter ainsi le recours philosophique au sens commun sous sa forme insuffisamment réfléchie:

Soit la thèse philosophique $\mathrm{T}$ et soit la thèse de sens commun U. Si U et $\mathrm{T}$ se contredisent directement ou indirectement, alors on doit tenir $T$ pour fausse.

La faute de raisonnement (bien connue) tient au fait que la conclusion qui s'impose, si U et T se contredisent, est que si ces propositions sont susceptibles d'être vraies ou fausses, ou bien $T$ est fausse et $U$ est alors vraie, ou bien $\mathrm{U}$ est fausse et $\mathrm{T}$ est alors vraie. En d'autres termes, lorsqu'il y a une contradiction entre une thèse philosophique et une proposition de sens commun, ce n'est pas nécessairement la thèse philosophique qui est fausse. Par exemple, si dire avec Galilée que la Terre est en mouvement, cela contredit le sens commun, alors ce n'est pas nécessairement Galilée qui a tort. 
Pour éviter la faute de raisonnement, on doit introduire une clause supplémentaire $\mathrm{V}$.

$\mathrm{V}: \mathrm{U}$ est vraie.

Étant donné que V, si $\mathrm{U}$ et $\mathrm{T}$ se contredisent directement ou indirectement, alors on doit tenir $\mathrm{T}$ pour fausse.

Maintenant, nous avons formulé l'ambiguïté fondamentale du recours philosophique au sens commun: si on a recours à $U$ pour rejeter $\mathrm{T}$, est-ce parce que $U$ est une proposition de sens commun ou parce que $U$ est une proposition vraie? On dissipera l'ambiguité en répondant que c'est parce que $U$ est une proposition vraie.

Évidemment, on pourrait être tenté de mettre tout le monde d'accord en disant que le recours au sens commun est un recours à des vérités de sens commun. Mais ce serait maintenir un flou artistique assez suspect, car il faut bien répondre clairement à la question: qu'est-ce qui compte, que ce soit des vérités ou qu'elles soient de sens commun? Si l'on parle de vérités du sens commun, il convient d'introduire un ordre de priorité entre "vérités" et «de sens commun».

Ce qui compte au premier chef, c'est que ce soit des vérités. Sans cela, le recours philosophique au sens commun est nécessairement sophistique.

Ce qui compte secondairement (car c'est sous la condition précédente), c'est que ces vérités soient de sens commun. Être de sens commun, pour une vérité, c'est une certaine manière d'être donnée. Une vérité est de sens commun lorsqu'elle est constamment présupposée, indéfectible, indéracinable, irrésistible, évidente, axiomatique. Cette liste hétéroclite laisse entendre que les modalités du sens commun sont très diverses et qu'il y a plusieurs manières possibles de concevoir un accès à des principes de base, selon qu'on insiste sur l'évidence d'une intuition, ou sur la nécessité logico-pragmatique d'une présupposition, ou encore sur la naturalité d'une croyance.

J'ai fait une distinction très forte entre «être une vérité » et "être de sens commun ", et j'ai soutenu que la première qualité ne dépend pas de la seconde. Cette distinction est nécessaire pour comprendre que pour que quelque chose soit une "vérité de sens commun ", ce qui est d'abord requis c'est que ce soit une vérité, et que cette question n'est pas réglée par le fait que c'est une proposition du sens commun.

Dans le premier temps de cet exposé, à la suite de Noah Lemos, j’ai proposé une formulation correcte du recours philosophique au sens commun, ou plus exactement de la condition nécessaire de la correction du recours philosophique au sens commun. Cette condition nécessaire est que les thèses de sens commun constituent des connaissances, selon un usage du terme qui exclut qu'une connaissance puisse être fausse. Cette condition suffit-elle à assurer la correction du recours philosophique au sens commun? Je ne le crois pas, mais cela n'est pas directement l'objet de cet exposé. Toutefois, c'est sans doute ce genre d'insuffisance que Collingwood 
avait à l'esprit lorsqu'il a élaboré sa critique de Moore et plus généralement celle du recours au sens commun. Il convient de se tourner maintenant vers les deux principaux arguments que Collingwood a élaborés contre Moore.

\section{Une première critique de Collingwood contre la forme mooréenne de l'argument du sens commun}

Dans An Essay on Philosophical Method (1933), Collingwood critique une forme de scepticisme qu'il appelle "philosophie analytique" parce qu'il s'agit notamment de cette philosophie qui, avec Moore, s'est donné pour tâche de décomposer les propositions en leurs constituants - il s'agit de la philosophie analytique dans le sens originel de l'expression. Cette philosophie est "sceptique" en un sens assez particulier. Elle n'est pas sceptique à l'égard de notre expérience du monde, mais à l'égard de la philosophie ellemême et spécialement de la métaphysique idéaliste. Elle a recours au sens commun comme à un garde-fou, alors même que le sens commun est usuellement l'antidote du scepticisme.

Pour clarifier ces usages du terme «scepticisme », il serait peut-être utile de faire une distinction entre deux scepticismes, celui qui est apparenté à l'idéalisme subjectif ou aux philosophies de la représentation (appelons-le "scepticisme moderne $»^{2}$ ), et celui qui consiste en une défiance à l'égard de la rationalité philosophique. Les philosophes du sens commun, qu'il s'agisse de Thomas Reid et de ses disciples ou bien de Moore, combattent le «scepticisme moderne" en s'appuyant sur le sens commun ${ }^{3}$. Sont-ils sceptiques au sens du scepticisme à l'égard de la philosophie?

Dans la présentation que donne Collingwood des philosophes du sens commun - il songe en particulier à Moore, dont il cite la "Defence of Common Sense » -, la définition du scepticisme (le scepticisme à l'égard de la philosophie) qui est retenue est la suivante: est sceptique la thèse selon laquelle la philosophie ne peut pas parvenir à construire rationnellement ses positions; une espèce particulière de scepticisme en conclut que la tâche de la philosophie est seulement critique, déconstructrice; une autre espèce de scepticisme en conclut que la philosophie est capable d'un résultat positif, toutefois elle ne parvient pas à ce résultat par ses propres forces, mais seulement en s'appuyant sur la science et sur le sens commun. L'important est de comprendre la distinction que propose Collingwood entre trois réponses possibles à la question suivante : les questions philosophiques sont-elles susceptibles de recevoir des réponses, et de quelle nature? Une première réponse

2. Le «scepticisme moderne" soutient sous une forme ou sous une autre la thèse selon laquelle l'esprit percevant ou connaissant n'a d'accès direct qu'à ses propres états mentaux ou bien n'a d'accès à une autre chose qu'à ses propres états mentaux qu'à travers la représentation qu'ils en donnent.

3. Pour une discussion du lien entre recours au sens commun et critique du scepticisme dans la philosophie britannique au XvIII ${ }^{\mathrm{e}}$ siècle, voir mon étude: "Les recours philosophiques au sens commun dans les Lumières britanniques ». 
est que les questions philosophiques sont susceptibles de recevoir des réponses philosophiques. C'est la réponse de Collingwood. Les deux autres réponses sont sceptiques. La deuxième réponse est que les questions philosophiques ne sont pas susceptibles de recevoir de réponses. Une troisième réponse est que les questions philosophiques sont susceptibles de recevoir des réponses, néanmoins ces réponses reposent non pas sur les ressources propres de l'argumentation philosophique, mais sur la science et sur le sens commun, en somme sur la rationalité savante et sur la rationalité commune en tant qu'elles sont extérieures à la rationalité philosophique.

Selon cette troisième réponse, les questions philosophiques ne peuvent pas être résolues par la philosophie elle-même et sont par conséquent de deux sortes: soit ce sont des questions qui peuvent être résolues par la science ou par le sens commun, soit, lorsqu'elles ne reçoivent pas de réponse de la science ou du sens commun, ce sont des questions qui ne peuvent pas être résolues du tout, qui sont proprement insolubles. De la première sorte relève la question de l'existence du monde matériel ou encore la question de l'existence des autres esprits. C'est le sens commun et non la philosophie qui répond à ces questions. De la seconde sorte relèvent les questions de l'existence de Dieu, de la vie future, ou encore de la nature de l'univers pris comme un tout. Le sens commun n'y répond pas (p. 141) - ici le sens commun est sans doute identifié à la science qui s'en tient à l'expérience.

La tâche de la philosophie est seulement "d'analyser la connaissance que nous possédons déjà ». Les propositions telles que «il y a un monde matériel» ne sont pas produites par la philosophie, mais données par la science ou par le sens commun (en l'occurrence par le sens commun), et le travail philosophique consiste dans une analyse ( «révéler la structure logique » des propositions), c'est-à-dire dans un éclaircissement sémantique ( «montrer ce que nous voulons dire exactement quand nous disons, par exemple, qu'il y a un monde matériel»).

Une question que Collingwood pose alors au philosophe analytique est de savoir quelle sorte de propositions il accepte d'inclure dans ce qui est sa position philosophique. Il pose au philosophe analytique la question de la conception de la philosophie, non pas entendue comme la question «qu'estce que la philosophie?", mais comme la question "quelle est votre position philosophique, qu'est-ce que vous mettez dans votre philosophie?».

Dans sa doctrine, le philosophe analytique mettra au moins deux ingrédients :

1) Des propositions de base qui ont été à tort critiquées ou écartées par d'autres philosophes, mais qui doivent être manifestement admises en tant que ce sont des propositions du sens commun. Bien que ces propositions constituent des données préphilosophiques de l'analyse, en somme une connaissance antérieure à la philosophie (par exemple «il y a un monde matériel »), elles font partie de la doctrine philosophique parce qu'elles 
font partie de ce qui distingue cette doctrine d'une autre doctrine, par exemple de l'idéalisme subjectif.

2) Des propositions qui sont non pas les données préphilosophiques de l'analyse, mais des résultats de cette analyse et qui ne sont donc pas des propositions de sens commun, mais l'éclaircissement ou l'anatomie philosophique de ces propositions. Par exemple, la proposition de sens commun "ceci est une main humaine" peut être analysée de telle manière que la proposition suivante en rend compte: «Il existe une et une seule chose dont il est vrai à la fois que c'est une main humaine et que cette surface est une partie de sa surface.»

Ici, Collingwood cite simplement l'article «Defence of Common Sense » de Moore (1925). L'exemple «ceci est une main humaine", dans ce contexte, ne renvoie pas à la prémisse fameuse de la preuve de l'existence du monde extérieur dans l'article «Proof of an External World» qui est bien postérieur (1939), mais aux pages 53-55 de l'article de 1925. "Ceci est une main humaine » est un exemple de propositions plus simples et plus difficiles à analyser que des propositions telles que "les choses matérielles existent ». La question de la nature des choses matérielles se joue dans l'analyse de ces propositions plus simples. Cette analyse a recours à la distinction entre la chose et des constituants qui sont ce que nous percevons d'elle, à savoir les sense-data (ici, cette surface perçue). Dans l'article de 1939, l'exemple «voici une main » et «en voici une autre» jouera un tout autre rôle puisque ce sera un exemple, non pas de propositions simples dont une analyse serait requise, mais de connaissance immédiate dont l'évidence est plus forte que les doutes à l'égard de l'existence des objets extérieurs.

Revenons à l'argumentation de Collingwood. Une doctrine analytique doit contenir deux classes de propositions: 1) Les propositions de sens commun qui sont à analyser, ou encore les données de l'analyse. 2) Les propositions philosophiques qui rendent compte de ces propositions de sens commun, les résultats de l'analyse. Mais selon Collingwood il faut ajouter une troisième classe de propositions qui sont implicitement contenues dans la position philosophique: les principes logiques et métaphysiques qui gouvernent cette analyse. Notamment, le principe logique selon lequel une proposition complexe peut être décomposée en propositions plus simples; le principe métaphysique selon lequel «les sense-data ne sont pas des entités mentales qui représentent d'une certaine manière les objets physiques mais sont réellement des parties des objets physiques ". C'est ce qui les distingue des idées de Descartes, de Locke, et de leurs successeurs. Par là, Moore ne souscrit pas au "scepticisme moderne».

Collingwood propose, si je puis dire, de faire le ménage dans le contenu de la position philosophique du "philosophe analytique » et de distinguer les propositions qui peuvent légitimement prétendre à en faire partie. 
Cela revient, devant une philosophie du sens commun, à chercher à déterminer ce qui, en elle, est vraiment de la philosophie.

Pour ce qui est de la première sorte de propositions, les données du sens commun, dans la mesure où la philosophie n'a pour tâche ni de les critiquer ni de les produire, mais seulement de les analyser, sont des données non philosophiques qui ne peuvent faire partie de la position philosophique elle-même. Les inclure dans la position philosophique serait contredire l'exigence d'un recours au sens commun en tant qu'il est extérieur et préalable à la philosophie.

Quant à la seconde sorte de propositions, les résultats de l'analyse, on aurait tort de croire qu'ils sont proprement philosophiques, puisque «l'analyse d'une proposition de sens commun établit ce que cette proposition signifie exactement; et si le datum de l'analyse est une proposition de sens commun, son résultat, qui, lui, est identique dans sa signification, est aussi une proposition de sens commun». Collingwood ne veut pas dire que la description «il existe une et une seule chose dont il est vrai à la fois que c'est une main humaine et que cette surface est une partie de sa surface » est une proposition de sens commun. Elle ne l'est manifestement pas. Mais dans la mesure où cette proposition donne la signification ou le résultat de l'analyse de la proposition de sens commun «ceci est une main humaine", elle ne nous donne rien de bien différent de ce que signifie la proposition de sens commun (du moins à supposer que l'analyse soit correcte). Les deux propositions ne sont pas de même niveau au sens où l'une est l'objet de l'analyse et l'autre est son résultat, mais elles sont cependant de même niveau au sens où le résultat n'est rien de plus que la formulation de la signification de la donnée initiale.

C'est seulement la troisième sorte de propositions qui peuvent légitimement prétendre à faire partie de la position philosophique: «les principes que suit l'analyse ». "Ces principes constituent une théorie sur la nature et la méthode de la philosophie; c'est une théorie philosophique, et même une théorie constructive» (p. 144-145). Or le philosophe analytique est un philosophe sceptique, de la seconde espèce, celle qui ne réduit pas la tâche de la philosophie à la partie déconstructrice, mais qui n'admet pas que la philosophie soit d'elle-même constructrice. Cette philosophie prétend donc "ne pas avoir de théorie constructrice ou systématique propre ». On comprend mieux pourquoi la philosophie du sens commun ne met pas en avant les principes qui régissent l'analyse des données du sens commun. C'est que ces principes constituent manifestement les éléments d'une "théorie constructrice ou systématique propre ", ce qui revient à "transgresser les règles » que le scepticisme à l'égard de la philosophie s'est fixées. Ce scepticisme n'a pas le courage d'assumer, ou d'avouer, ses principes logiques et métaphysiques, et encore moins leur caractère systématique.

Cette critique de la philosophie du sens commun s'adresse principalement à Moore et suppose que le recours au sens commun est suivi d'une 
analyse des propositions de sens commun. Il est douteux que cette critique puisse porter de manière pertinente contre la première école du sens commun, celle de Reid et des Écossais, dans la mesure où cela supposerait que cette école pratiquait une analyse de ce type, ce qui n'est pas le cas.

$\mathrm{Si}$ on se reporte à la formulation de l'argument du sens commun par laquelle j'ai commencé, on note que la critique de Collingwood est assez indirecte.

$\mathrm{U}$ est une proposition du sens commun.

$\mathrm{T}$ est une thèse philosophique.

$\mathrm{V}: \mathrm{U}$ est vraie.

Étant donné que $\mathrm{V}$, si $\mathrm{U}$ et $\mathrm{T}$ se contredisent directement ou indirectement, alors on doit tenir $\mathrm{T}$ pour fausse.

Dans cette première critique, Collingwood n'attaque pas frontalement cet argument. Mais il indique un défaut dans son principe. Ce qui ne va pas ici dans ce recours au sens commun, c'est que la philosophie prétend aller chercher dans des propositions préphilosophiques du genre de U de quoi ironiser à l'égard de la philosophie elle-même, et spécialement de l'idéalisme. Mais cette philosophie sceptique, qui prétend ne pas aller plus loin que l'analyse des propositions du genre de $\mathrm{U}$, repose en réalité sur des principes logiques et métaphysiques qui ne sont pas du tout du genre de U.

\section{Une seconde critique de Collingwood contre l'argument du sens commun en général}

Dans le Nouveau Léviathan (1942), on trouve une discussion de la philosophie du sens commun qui adopte un autre angle d'attaque et qui, si elle garde toujours en ligne de mire la conception mooréenne de la philosophie analytique, est d'une portée plus générale. À ma connaissance, ce second argument n'a pas retenu l'attention ${ }^{4}$.

On a noté que dans An Essay on Philosophical Method le thème de Collingwood est à trois termes: la philosophie, la science et le sens commun. Dans le Nouveau Léviathan, nous avons affaire à un thème à deux termes: la science et le sens commun. Dans la première critique, la science est mise sur le même pied que le sens commun (au point que la discussion de Moore, on l'aura remarqué, laisse la science de côté au profit du seul sens commun) et est traitée comme une autorité extérieure à la philosophie. Dans la seconde critique, celle du Nouveau Léviathan, la science, identifiée à la connaissance (qu'elle soit spéculative ou expérimentale), relève tantôt

4. La partie la plus visible de la critique de Moore par Collingwood, dans l'ensemble des documents réunis par David Boucher dans son édition de The New Leviathan, se trouve dans les conférences sur "Goodness, Rightness, Utility", a pour cible les Principia Ethica et tourne par conséquent autour de la question de l'intuitionnisme moral et non directement de l'argument du sens commun. 
du sens commun (quand il s'agit d'un état passé de la science, qui constitue une connaissance d'arrière-plan), tantôt de la connaissance vivante, ce qui inclut la philosophie. Je signale ce point uniquement pour prendre acte d'une différence manifeste entre ces deux critiques et pour attirer l'attention sur le fait que cette différence ne doit pas masquer leur profonde unité d'inspiration.

C'est dans le premier chapitre du Nouveau Léviathan que cette critique est formulée, dans une sorte de digression dont l'occasion est fournie par l'examen de la question «qu'est-ce que l'homme? ». Dans l'examen de cette question, Collingwood signale qu'elle a évidemment des rapports avec la question «qu'est-ce que la matière? " et la question «qu'est-ce que la vie? ", puisque l'homme est à la fois esprit et corps. Mais l'objet du Nouveau Léviathan, l'étude de la communauté et de la civilisation, concerne l'aspect sous lequel l'homme est esprit. C'est pourquoi les questions de la matière et de la vie, bien qu'elles soient pertinentes lorsqu'il s'agit de comprendre ce qu'est le corps de l'homme, doivent être laissées de côté. À ce point de son raisonnement, Collingwood indique ce qu'il répondrait à la question «qu'est-ce que la matière? » et à la question «qu'est-ce que la vie ? ", bien que ces questions n'appellent pas de réponse, j'y insiste, dans le contexte du Nouveau Léviathan. On pourrait s'attendre à une réponse de sens commun. Collingwood formule lui-même cette réponse de sens commun en l'entourant de guillemets, comme il le fait souvent dans cet ouvrage: «La physique et la chimie, dira-t-il [un lecteur] peut-être, c'est la science de la matière; et tout le monde sait ce qu'est la matière. La physiologie est la science de la vie; et tout le monde sait ce qu'est la vie ${ }^{5}$ (1.41). Nous savons communément ce qu'est la matière et nous savons communément ce qu'est la vie. Les sciences telles que la physique et la chimie sont des sciences de ces choses dont nous savons communément ce qu'elles sont. Cette réponse est profondément fausse. Il faut remettre les choses à l'endroit. À la question «qu'est-ce que la vie? » il faut répondre "c'est ce qu'étudie la physiologie». Car celui qui s'efforce de savoir ce qu'est la vie et qui à cette fin fait de la physiologie a une définition (rudimentaire s'il est un débutant; plus élaborée s'il est un expert) de la science qu'il pratique, et non de l'objet de cette science (1.43). Il n'a pas de définition de l'objet de la science indépendamment de cette science. Pour le physiologiste, quel que soit son degré d'avancement dans cette science, "c'est la nature de la physiologie qui est relativement certaine, la nature de la vie qui est relativement vague » (1.47). Cette thèse de Collingwood, qui affirme que nous ne pouvons pas identifier l'objet d'une science indépendamment de cette science ou extérieurement à cette science, pourrait justifier la qualification d'idéalisme, du moins si on prendre garde

5. Pour le Nouveau Léviathan, j’utilise la traduction de Lucien Carrive. Les références sont données selon la numérotation qu'emploie Collingwood, de telle sorte que le lecteur peut se référer également à l'édition de l'original anglais par David Boucher. 
de noter qu'il ne s'agit pas du tout ici d'un idéalisme subjectif, qui réduirait l'être de l'objet au savoir du sujet. Collingwood ne dit pas que la vie n'est rien en dehors de la physiologie, mais qu'il n'y a pas de réponse à la question "qu'est-ce que la vie? " en dehors de questions que la physiologie pose à la vie. Il me semble que cette thèse est implicitement liée à ce que Collingwood appelle sa nouvelle logique, celle qui affirme que les propositions doivent être considérées à partir des questions par rapport auxquelles elles sont des réponses.

Une conséquence particulière est que la différence entre le débutant dans une science et le maître dans cette science ne peut pas être formulée de la manière suivante: le débutant est celui qui ne connaît pas bien le sujet de l'étude et qui n'en sait que ce que sa maigre science lui en dit, tandis que le maître est celui qui connaît à fond ce qu'est le sujet de son étude et n'a plus rien à attendre de la science à cet égard. Collingwood soutient au contraire que le maître est exactement dans la même position que le débutant, c'est-àdire que tout ce qu'il sait de l'objet de la science consiste dans l'état de sa science. Il est un expert dans cette science, et c'est dans les termes de cette science que l'objet est définissable. Le débutant espère que la science découvrira enfin la vraie nature de l'objet et qu'elle sera alors science de cet objet, définissable dans les termes de cet objet (la physiologie accomplie serait la connaissance de ce qu'est vraiment la vie), mais ne plus être un débutant, c'est avoir compris que c'est toujours la vie qui est définissable en termes de physiologie, jamais l'inverse. Je cite 1.46:

Un homme cesse d'être un débutant dans une quelconque science donnée et il devient un maître dans cette science quand il a appris que ce renversement attendu n'arrivera jamais et qu'on restera un débutant toute sa vie.

Si on prend l'exemple de la discussion sur l'enseignement du dessein intelligent, une réponse collingwoodienne pourrait être simplement que c'est une profonde erreur que de penser que la science issue de Darwin et l'enseignement créationniste sont en concurrence à propos du même objet, comme si cet objet - la vie et plus précisément la diversité des formes vivantes - pouvait être défini hors de la science.

Je viens de reconstituer l'erreur que constitue la thèse selon laquelle on pourrait définir l'objet d'une science grâce à une connaissance de sens commun de cet objet qui serait indépendante de cette science. Pour Collingwood, ce n'est pas seulement une erreur, mais une faute très dangereuse: "Croire que la physique ou la chimie devraient être définis en termes de matière ou la physiologie en termes de vie est plus qu'une erreur insigne; c'est une menace contre l'existence de la science » (1.51). Pourquoi est-ce une faute très dangereuse? Parce que c'est dogmatique, autoritariste, et obscurantiste.

C'est à ce point de son raisonnement que Collingwood attaque ce que je propose d'appeler dorénavant l'argument du sens commun. Je distingue entre la notion du sens commun et l'argument du sens commun de la manière 
suivante: la notion du sens commun est l'idée de l'existence d'une connaissance commune, non technique, antérieure à la connaissance scientifique élaborée et présupposée par elle; l'argument du sens commun est un usage très particulier de cette notion du sens commun. L'argument consiste à employer la notion comme un critère ou un canon, voire comme un organon de la connaissance scientifique. Il conviendrait, d'ailleurs, de restreindre l'usage de l'expression "philosophie du sens commun " aux seuls cas des philosophies qui s'appuient sur l'argument, et pas seulement sur la notion. Une formulation de l'argument du sens commun peut être la suivante: étant donné qu'il existe une connaissance commune, non technique, antérieure à la connaissance scientifique élaborée et présupposée par elle (ce qu'établit la notion du sens commun), si un élément de la connaissance scientifique contredit directement ou indirectement cette connaissance commune, alors cet élément doit être rejeté, et si la connaissance scientifique comporte nécessairement cet élément, alors c'est tout le corps de la connaissance scientifique qui doit être rejeté.

Or Collingwood a formulé avec précision ce prétendu argument:

Si quelque chose, quoi que ce soit, que les scientifiques s'imaginent avoir découvert sur la matière ou sur la vie est incompatible avec quoi que ce soit que contienne ou qu'implique cette connaissance non scientifique ou préscientifique, les scientifiques ont fait une erreur (1.53).

Nous reconnaissons ici la formulation du recours philosophique au sens commun qui a été explicitée de la manière suivante:

$\mathrm{U}$ est une proposition du sens commun.

$\mathrm{T}$ est une thèse philosophique.

$\mathrm{V}: \mathrm{U}$ est vraie.

Étant donné que $\mathrm{V}$, si $\mathrm{U}$ et $\mathrm{T}$ se contredisent directement ou indirectement, alors on doit tenir $\mathrm{T}$ pour fausse.

Collingwood estime que la proposition $\mathrm{V}$ est parfaitement dogmatique tant que sa vérité est attestée par le sens commun et non par la connaissance scientifique vivante (ce qui inclut ici la philosophie). Dans l'attente, on n'a pas plus de raisons de rejeter T qu'on a de raisons de rejeter U. Bien plus, l'usage qu'on fait des propositions du sens commun contre les thèses philosophiques est suspect parce qu'il suppose que nous serions dotés d'un meilleur mode de connaissance que la connaissance scientifique vivante. C'est une faute impardonnable.

Les paragraphes 1.51-57 du Nouveau Léviathan déclinent les conséquences de la faute impardonnable:

1. Cela justifie la misologie, puisque le recours philosophique au sens commun suppose qu'il n'est pas besoin d'étudier la science de la matière pour savoir ce qu'est la matière (1.51). 
2. Cela justifie l'obscurantisme et la haine du progrès, puisqu'on suppose que la connaissance de sens commun est parfaite et n'a pas à être rectifiée (1.52).

3. Cela justifie que l'on écarte les méthodes expérimentales si leurs résultats contredisent les convictions pré-expérimentales (1.54).

4. Cela justifie que l'on écarte les méthodes logiques si leurs résultats contredisent les convictions pré-logiques (1.55).

5. Cela justifie la «persécution des scientifiques» (1.57), particulièrement lorsqu'ils osent aller contre les prétendues connaissances de sens commun :

D'un seul coup, en énonçant la proposition apparemment inoffensive que la physique ou la chimie est la science de la matière, la physiologie est la science de la vie, et autres semblables, nous avons évoqué l'appareil entier de la persécution scientifique, je veux dire de la persécution des scientifiques pour avoir osé être des scientifiques ${ }^{6}$.

Il ne faudrait cependant pas en conclure que Collingwood rejette la notion de sens commun et lui dénie toute portée philosophique. Je souscris à la remarque de Guiseppina D'Oro, selon laquelle "for Collingwood, philosophical analysis stands in a rather complex relation to common sense ${ }^{7}$. Cependant, alors que G. D'Oro caractérise la complexité de cette relation en situant l'attitude de Collingwood à égale distance de la défiance cartésienne à l'égard du préjugé et de l'usage thérapeutique et antithéorique du recours à l'ordinaire, il me semble qu'on peut aussi comprendre la manière dont Collingwood conçoit la relation entre philosophie et sens commun en utilisant la distinction que j'ai proposée entre notion du sens commun et argument du sens commun. En somme, Collingwood critique l'argument au nom d'une caractéristique de la notion. En effet, une caractéristique importante de cette notion, pour Collingwood, est son historicité. C'est précisément ce qui interdit de donner au sens commun le rôle d'un repère intangible ou d'un stock immuable de connaissances. C'est cet aspect que je vais examiner maintenant.

6. En 5.31-33, Collingwood reprend l'expression de 1.57 et mentionne l'article de Moore, "The Refutation of Idealism» (1903, repris en 1922 dans les Philosophical Studies). Il écrit que Moore et les «nombreux philosophes contemporains qui sont de la même opinion [allusion probable aux réalistes d'Oxford, tel John Cook Wilson] enseignent une attitude de soumission" à l'égard du sens commun. Le recours à cette faculté les dispense de donner les raisons de leurs thèses spéculatives.

7. G. D’Oro, «Collingwood on Philosophical Knowledge and the Enduring Nature of Philosophical Problems », p. 108. 


\section{La notion du sens commun et les présuppositions absolues}

La nature du sens commun est historique. Ce point est suggéré par l'exemple que donne Collingwood dans le Nouveau Léviathan (1.58-60): le sens commun qu'on oppose aux innovations de la science vivante est en réalité, non pas une connaissance préscientifique, mais une connaissance postscientifique, c'est-à-dire un état dépassé de la science. Une nouvelle doctrine est persécutée au nom d'une ancienne doctrine. "Ce qui en profite en réalité, ce fut toujours la science dépassée » (1.58). Cette idée que le sens commun est une sorte de passé de la science, ou de la science passée, est profondément étrangère aux philosophies du sens commun.

Il est vrai que, dans ce contexte, Collingwood est en train de discuter l'argument du sens commun et non d'exposer positivement la notion du sens commun. Il dénonce le recours au sens commun comme asile de l'ignorance. Cependant, je crois que Collingwood lui-même, lorsqu'il donne un statut positif à la notion de sens commun, souligne son caractère historique.

Pour confirmer ce point, il suffit de chercher ce qui, dans la pensée de Collingwood et en dehors du contexte de la réfutation des défenseurs du sens commun, est la formulation la plus exacte de la notion du sens commun, c'est-à-dire de l'idée de l'existence d'une connaissance commune, non technique, antérieure à la connaissance scientifique élaborée et présupposée par elle. Il me semble qu'on trouve cette formulation avec l'idée de présupposition, telle qu'elle est élaborée notamment dans An Essay on Metaphysics (1940).

Collingwood distingue entre les présuppositions relatives et les présuppositions absolues. Considérons les questions Q1 et Q2 et leurs réponses respectives $\mathrm{R} 1$ et $\mathrm{R} 2$. Une présupposition est relative lorsqu'elle est la relation entre une question $\mathrm{Q} 2$ et une réponse $\mathrm{R} 1$, de telle nature que la question Q2 n'aurait pas été posée et n'aurait pas pu recevoir la réponse R2 si une réponse $\mathrm{R} 1$ n'avait pas été donnée à une autre question antérieure $\mathrm{Q} 1$. Une thèse de Collingwood, souvent présentée comme sa logique des questions et des réponses, est que la signification d'une proposition ne peut pas être correctement appréhendée en dehors de cette suite de présuppositions. Par exemple, la proposition «Pierre n'a pas fait les courses au supermarché » est implicitement une réponse à la question «est-ce que Pierre a fait les courses au supermarché ?"; cette question a plusieurs présuppositions: qu'il existe un supermarché dans lequel Pierre peut faire les courses et que plus généralement il est capable de les faire, mais aussi, sans doute, que Pierre devait faire les courses, etc. Ces présuppositions elles-mêmes sont implicitement en rapport avec des questions telles que "existe-t-il un supermarché dans les environs? » ou "Pierre a-t-il les moyens de se déplacer?». Les présuppositions relatives sont des réponses, susceptibles d'être vraies ou fausses, à ces questions.

Une présupposition est relative lorsqu'elle rend une question Q2 possible et lorsqu'elle est elle-même une réponse R1, susceptible d'être vraie ou 
fausse, à une question antérieure Q1. Une présupposition est absolue lorsqu'elle rend une question possible sans être elle-même une réponse, susceptible d'être vraie ou fausse, à une question fondamentale. Collingwood appelle métaphysique la science des présuppositions absolues.

Giuseppina D'Oro a insisté sur le fait que les présuppositions absolues sont des croyances de base, non pas au sens où on ne saurait psychologiquement y échapper, mais au sens où elles sont logiquement nécessaires ${ }^{8}$. Il me semble qu'on retrouve ici une caractéristique importante du sens commun, du moins dans la conception qu'en avait Reid, et que les présuppositions absolues de Collingwood, en-deçà desquelles on ne saurait remonter, sont, à cet égard, comparables aux premiers principes de Reid. Celui-ci prend notamment les exemples du principe de causalité ou du principe selon lequel une qualité exige un sujet qui la supporte. Les premiers principes sont des conditions fondamentales de la connaissance et non pas des croyances naturelles au sens du naturalisme humien, c'est-à-dire des croyances dont on ne peut psychologiquement se défaire. G. D'Oro distingue également les présuppositions absolues de premiers principes "au sens du cartésianisme ", c'est-à-dire de principes qui seraient l'objet d'une connaissance intuitive. Mais là encore, même s'il arrive à Reid de parler d'intuition à propos de l'accès aux premiers principes, cet accès consiste en une régression vers les présuppositions - pragmatiques autant qu'épistémiques - de notre activité ordinaire de connaissance ${ }^{9}$. Ce que Reid appelle "premiers principes» correspond à des conditions de possibilité non seulement de la science mais de la vie commune, qu'atteste la moindre conversation et vers lesquelles on remonte dialectiquement ${ }^{10}$. La comparaison entre les présuppositions absolues de Collingwood et la notion reidienne de sens commun n'est donc pas injustifiée. D'Oro rapproche Collingwood du kantisme, en insistant sur une conception des présuppositions qui se distingue autant du psychologisme humien que de l'intuitionnisme cartésien, mais il me semble que ce «ni Descartes, ni Hume» était également le mot d'ordre du projet reidien.

8. G. D’Oro, «Collingwood on Philosophical Knowledge and the Enduring Nature of Philosophical Problems", p. 98.

9. Essays on the Intellectual Powers, I, 2, p. 40-41: «Ce sont des principes qui, bien qu'ils n'aient pas le même genre d'évidence que les axiomes mathématiques, ont cependant une évidence telle que tout homme doté de l'entendement commun leur donne promptement son assentiment et trouve qu'il est absolument nécessaire qu'il conduise ses actions et ses opinions par leur moyen, dans les affaires ordinaires de la vie.»

10. Je renvoie à un seul exemple, à propos du premier principe selon lequel toute qualité requiert un sujet, ibid., p. 43 : «Si une personne estimait qu'il est pertinent de nier que ces choses [figure, couleur, dureté, douceur, etc.] sont des qualités ou qu'elles requièrent un sujet, je l'abandonnerais à son opinion comme une personne qui nie les premiers principes et avec laquelle on ne saurait raisonner. Si elle a du bon sens, elle trouvera qu'elle ne peut converser une demi-heure sans dire des choses qui impliquent le contraire de ce qu'elle prétend croire. » 
Est-il utile de le préciser? Rapprocher la notion collingwoodienne de sens commun de celle que proposait Reid, ce n'est en rien suggérer que Collingwood souscrirait à l'argument du sens commun tel que le formulait Reid.

Quoi qu'il en soit, une différence très importante entre Reid et Collingwood, sur cette question des présuppositions absolues, est que Reid maintenait le caractère naturel, anhistorique, éternel, du sens commun, tandis que Collingwood estime que les premiers principes sont des modèles historiques. Plus exactement, ce qui est de nature historique, c'est la notion de la causalité (la doctrine en quelque sorte, même si elle n'est pas élaborée philosophiquement) qui est présupposée par le style d'explication causale que pratique une communauté humaine. Selon Collingwood, les présuppositions absolues doivent être compossibles (il dit "consupponible », co-présupposables), elles sont des éléments de constellations de présuppositions. Elles sont surtout manifestement de caractère historique, non au sens où elles seraient de l'ordre de l'événement historique, mais au sens où elles constituent le paradigme d'une époque ou d'une société, à l'intérieur duquel les événements historiques sont susceptibles d'être compris. C'est pourquoi la métaphysique est une science historique. Je ne développe pas cet aspect de la pensée de Collingwood et je n'entre pas dans la controverse sur son historicisme. Il s'agissait principalement de signaler que sa critique de l'argument du sens commun ne l'empêche pas de maintenir ce que j'ai appelé la notion de sens commun.

Comme on le voit, si on appelle sens commun un ensemble de présuppositions absolues (il ne serait sans doute pas absurde de l'étendre à des présuppositions relatives générales), et si on souligne le caractère historique du sens commun, le recours au sens commun change profondément de portée et de signification. Il ne saurait s'agir d'un recours dogmatique destiné à clore toute question, mais au contraire, dans la mesure où c'est seulement le questionnement dialectique qui est susceptible de nous faire remonter jusqu'aux présuppositions absolues, le recours au sens commun suppose l'exercice même de l'interrogation métaphysique et historique. Ce type de recours est clairement compatible avec la réponse que Collingwood a donnée, dans An Essay on Philosophical Method, à la question «les questions philosophiques sont-elles susceptibles de recevoir des réponses, et de quelle nature?». En somme, contrairement à ce que croyait Moore, on ne saurait faire taire la philosophie au nom du sens commun, puisque le sens commun est ce que la philosophie doit interroger. 


\section{Bibliographie}

Collingwood, Robin G. An Essay on Philosophical Method, Oxford, Clarendon Press, 1933; réédition, Bristol, Thoemmes Press, 1996.

—. The New Leviathan, David Boucher dir., Oxford, Clarendon Press, 1992.

—. Le Nouveau Léviathan, trad. Lucien Carrive, Paris, Kimé, 2001.

—. An Essay on Metaphysics, Rex Martin dir., Oxford, Oxford University Press, 1998.

D’Oro, Guiseppina. "Collingwood on Philosophical Knowledge and the Enduring Nature of Philosophical Problems ", British Journal for the History of Philosophy, 12 (1), 2004, p. 93-109.

Jaffro, Laurent. «Les recours philosophiques au sens commun dans les Lumières britanniques ", dans Le Sens commun, Pierre Guenancia et Jean-Pierre Sylvestre, dir., Dijon, Éditions universitaires de Dijon, 2004, p. 19-45.

Lemos, Noah. Common Sense. A Contemporary Defense, Cambridge, Cambridge University Press, 2004.

Moore, George Edward. "The Refutation of Idealism» (1903), dans Philosophical Studies, G. E. Moore, Londres, Routledge \& Kegan, 1922, p. 1-30.

—_. «A Defence of Common Sense» (1925), dans Philosophical Papers, G. E. Moore, Londres, George Allen \& Unwin, 1959, p. 32-59.

—. "Proof of an External World ", Proceedings of the British Academy, 25, 1939, p. 273-300.

Reid, Thomas. Essays on the Intellectual Powers of Man, Derek R. Brookes dir., Édimbourg, Edinburgh University Press, 2002. 